Portland State University

PDXScholar

\title{
Acousto Characterization of Fluid-like Mesoscopic Films Under Shear
}

\author{
Rodolfo Fernandez \\ Portland State University \\ Xiaohua Wang \\ Portland State University \\ Andres H. La Rosa \\ Portland State University, andres@pdx.edu
}

Follow this and additional works at: https://pdxscholar.library.pdx.edu/phy_fac

Part of the Physics Commons

Let us know how access to this document benefits you.

\section{Citation Details}

R. Fernandez, X. Wang, and A. H. La Rosa, "Acousto Characterization of Fluid-like Mesoscopic Films under Shear," Proceedings of the Nanotechnology (IEEE-NANO) 11th IEEE International Conference, p. 903-906 (2011).

This Post-Print is brought to you for free and open access. It has been accepted for inclusion in Physics Faculty Publications and Presentations by an authorized administrator of PDXScholar. Please contact us if we can make this document more accessible: pdxscholar@pdx.edu. 


\title{
Acousto Characterization of Fluid-like Mesoscopic Films under Shear
}

\author{
Rodolfo Fernandez, Xiaohua Wang, and Andres La Rosa* \\ Department of Physics, Portland State University, Portland, OR 97201. \\ ${ }^{*}$ Corresponding author, email: $\underline{\text { andres @ pdx.edu }}$
}

\begin{abstract}
Full understanding of the physics underlying the striking changes - in viscoelasticity, relaxation time, and phase transitions-that mesoscopic fluid-like systems undergo when placed under confinement or when adsorbed at solid surfaces constitutes a long standing scientific challenge. One of the methods used to characterize these films consists of bringing a solid boundary closer to another solid boundary (while in relative lateral periodic motion) with a liquid trapped in between. In addition, using a tapered probe $(\sim 50 \mathrm{~nm}$ apex diameter) as one of the boundaries improves the lateral resolution of the measurement. In this scenario, the dynamics of the fluid is inferred from the changes in the tapered probe's motion. However, due to the complexity of the film's dynamics, different and sometimes conflicting experimental results are reported; in particular, for example, whether the motion of the probe changes due to its interaction with the fluid alone, or due to its intermittent mechanical contact with the solid substrate. Newer analytical methods would be highly desirable. Herein we report the monitoring of mesoscopic film dynamics from an acoustic measurements perspective (complemented with other more conventional sensing methods for control and comparison purposes). More specifically, two acoustic-based methods, Whispering-Gallery Acoustic Sensing or WGAS (that uses an acoustic sensor attached to a tapered probe) and Shearforce/Acoustic Near-field Microscopy or SANM (that uses an acoustic sensor attached to the solid substrate), monitor the effects that shear-force interactions exert not only on the laterally oscillating probe but also on the trapped mesoscopic fluid itself (as acoustic waves engendered at the fluid film couple into the static substrate and subsequently reaching the SANM acoustic transducer). One significant result of these measurements constitute the supporting evidence that the probe's motion is affected even when not in mechanical contact with the solid substrate, hence highlighting the role played by the adsorbed mesoscopic fluid layer as the source of the shear force interactions. On the other hand, to further support the SANM working principle (i. e. the measurement of acoustic waves engendered at adsorbed films of nanometer-sized) control experiments have also been performed for interrogating the dynamics of small millimeter-sized drops of water.
\end{abstract}

\section{INTRODUCTION}

Friction phenomena, comprising the interactions (of potentially multiple origins) between two solid surfaces in contact and sliding relative to each other, are not wellunderstood yet. It is not unusual to find that, at the macroscopic level, the subject is mostly tackled in a serendipitously manner. It is increasingly being accepted that a full understanding of friction may require studies from a mesoscopic scale perspective [1][2][3]. To reach such detailed resolution, one approach opts using a sharp probe as one of the solid boundaries, hence simplifying the problem to the friction interaction between a surface and a single nano-sized asperity. The study of friction has also witnessed progress with the development of theoretical models [4, 5, 6]. Indeed, numerical simulations [7], supported by experimental results [8], have shed lights into these studies; it appears that an important aspect of friction phenomena can be attributed to the mesoscopic fluid-like film found trapped between two sliding solid boundaries at typical ambient conditions. The interaction between the two solid sliding boundaries, mediated by the presence of trapped mesoscopic fluids, will be referred here as "shear-force" interactions. Given their now apparent important role as the source of shear-forces, herein we present preliminary results of a planned series of systematic measurements aimed at testing the dynamics of mesoscopic fluid-like films using a variety of metrology tools. The latter includes the conventional tuning-fork based scanning probe microscopy [9] and the newer acoustic-based probing technique that our laboratory has developed recently, namely Shear-force/Acoustic Nearfield Microscopy (SANM) [10] and Whispering-Gallery Acoustic Sensing (WGAS) [11].

The typical experimental setting to be considered here is a laterally oscillating tapered probe (attached to a $32 \mathrm{kHz}$ quartz tuning fork, or QTF, that drives the oscillations via electrical means) with its tip apex placed in the proximity of a flat solid boundary [9]. At ambient conditions both solid bodies have a mesoscopic fluid-like layer naturally adsorbed onto their surfaces, which mediate their shear-force interactions. As shown in Fig.1, to implement SANM an acoustic transducer is attached to the bottom of the flat solid substrate[10], while WGAS is implemented by simply attaching an acoustic transducer at the perimeter of the microscope frame (the exact position around the frame's perimeter optimized until attaining a maximum response.) [11]. The application of a thin layer of vacuum grease between the sample and the acoustic transducer improves the acoustic coupling. While a QTF monitors the effect that shear-force interactions have on the probe, SANM monitor the dynamic response of the trapped fluid film (through the detection of the acoustic wave engendered at the film, which couples into the solid substrate and subsequently reaches the SANM acoustic transducer). On the other hand, the acoustic WGAS signal provides a more accurate indication of the probe's oscillatory motion, compared to the QTF's electrical admittance (in the latter, the intrinsic capacitance of the QTF convolutes the electrical measurement). The acoustic 
measurements are complemented with the monitoring of the onset of tunnelling current (which should occur at the tipsubstrate mechanical contact). A combination of these techniques, all acquired simultaneously, will likely contribute to provide a clearer picture of the dynamics of mesoscopic fluids under shear.

\section{EXPERIMENTAL SETUP}

A schematic of the experimental arrangement is shown in Fig. 1. In addition to the combined SANM and WGAS setup, a gold tip is electrically biased (250 mV DC), and the eventual current between the sample and the tip is monitored by a current amplifier (SR570, Stanford Research Systems). The detection of the current is implemented via lock-in amplifier, hence emphasizing the detection of intermittent mechanical contact between the probe and the substrate. A gold coated (via sputtering) mica sheet was placed on top of the SANM acoustic sensor (SE40-Q, Dunegan Engineering Consultants, Inc.) The WGAS sensor is an acoustic transducer (3 mm diameter sensitive area SE 25-P 42, from DECI) positioned around the perimeter of the microscope's frame, which plays also the role of an acoustic cavity. The exact location of the sensor is determined by the different acoustic nodes where the WGAS signal reaches a maximum (such locations vary, depending on the QTF operating frequency)[11].

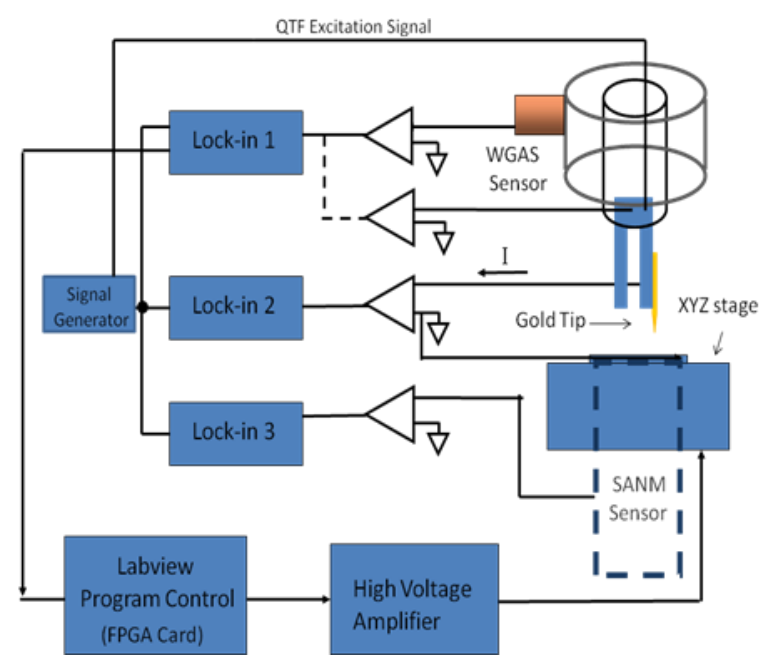

Fig. 1 WGAS and SANM experimental setup, complemented with the QTF's electrical admittance and the tunneling current measurement. All signals measured simultaneously and synchronously, with all the lock-in amplifiers referenced to the same operating frequency set by the signal generator.

The probe-sample distance is controlled by either $i$ ) moving the probe with a linear piezoelectric stage (OP65, Mad City Labs, equipped with strain gauge sensory feedback control to overcome piezoelectric effects) not shown in the figure, or ii) moving the sample with the Z-stage of a XYZ scanner (Tritor-100, Piezosystem Jena) equipped with capacitance feedback sensory to overcome piezoelectric hysteresis) as shown in the figure. Finally, the etched gold wire was mounted on a QTF and a copper wire completed the circuit electrically connecting the tip to the rest of the setup. All the signals were synchronously detected with the lock-ins referenced to a single signal generator, the latter also used to drive electrically the QTF. The time constant in all lock-in amplifiers was set to $30 \mathrm{~ms}$. The velocity at which the probe is approached towards the sample can be controlled by the operator.

As the probe approaches to, or retracts from, the substrate, several signals can be monitored simultaneously: the QTF's electrical admittance, the SANM and WGAS signals, and the tunneling current. In the results presented below, special emphasis is placed to the SANM sensor that monitors the efficiency at which the oscillations of the fluid layer couple into the substrate. An important point to discern is whether this coupling occurs even when the laterally oscillating solid tip does not make mechanical contact with the solid substrate. Monitoring simultaneously the tunneling current between the probe and sample helps to discern this aspect.

\section{RESULTS}

Fig. 2 shows approach and retraction traces acquired with the probe travelling vertically, towards to and away from, the sample at $1.5 \mathrm{~nm} / \mathrm{s}$. The zero coordinate of the graph's horizontal axis (the vertical z-axis in the experimental setup) has been chosen arbitrarily, but close to the position where the tunneling current starts to flow. The probe's initial amplitude of oscillation was $2 \mathrm{~nm}$ (estimated the QTF admittance measured when the probe was far away from the sample).

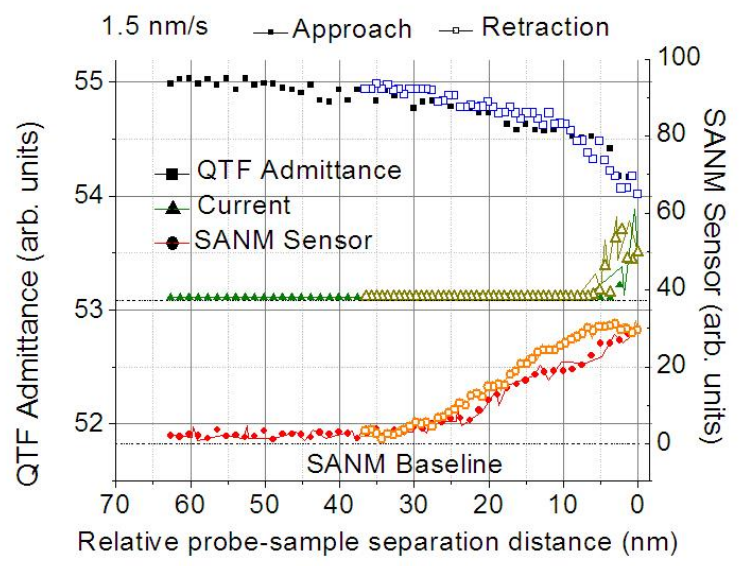

Fig. 2 Variations of the QTF admittance (top), acoustic SANM (bottom), and tunneling current (middle) as the probe (laterally oscillating at a fixed frequency) approaches (traces with filled makers) to and retracts (traces with open markers) from the sample. The zero base line of the tunneling current trace has been shifted upwards for clarity; the maximum detected current is $100 \mathrm{nA}$. Notice the correlation between the QTF and the SANM signals, and that they start to vary quite far away (at least $30 \mathrm{~nm}$ ) from the distance at which the unset of the tunneling current occurs.

Notice that during the approaching process the QTF signal starts to decrease slightly around the $30 \mathrm{~nm}$ mark. The SANM acoustic signal simultaneously starts to increase above the noise level, suggesting the phonon formation at the surface of the solid substrate. It is also observed that at the onset of QTF and SANM signals variations, no current is detected yet; i.e. the changes start when there is not mechanical contact between the solid probe and the solid 
substrate. As the probe keep approaching the sample, a definitely clearer change of the QTF and the SANM signals is measured at the $20 \mathrm{~nm}$ mark, while still registering a lack of current flow. As a final observation, there is a hysteresis effect reveled by the SANM approaching/ retraction traces (but not revealed, in this particular case, by the QTF signal). This hysteresis effect may provide useful information about the adhesive properties of the mesoscopic fluid layer.

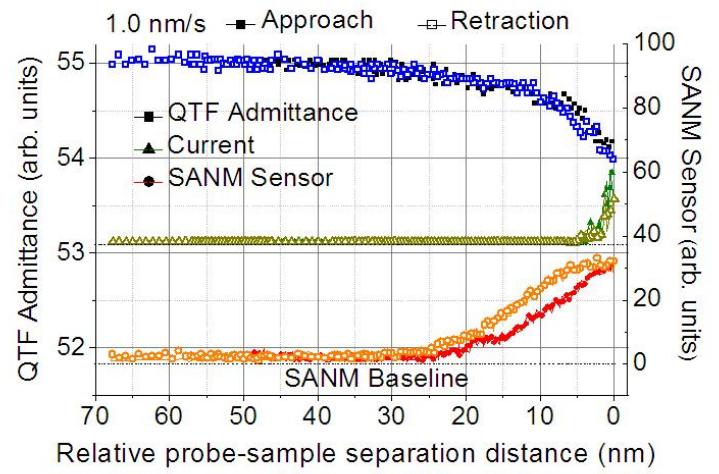

Fig. 3 Approach and retraction traces acquired under similar conditions as presented in Fig.2, except that the tip travelled at a $1 \mathrm{~nm} / \mathrm{s}$.

Fig.3 shows the reproducibility of the measurement, since it was obtained using the same procedure as the result presented in Fig.2 (same gold tip and same gold-coated mica sample), except for using a slower tip velocity $(1.0 \mathrm{~nm} / \mathrm{s})$. Notice the hysteresis displayed by the approaching/retraction traces of the SANM signal is reproduced once again, this time with more clarity.

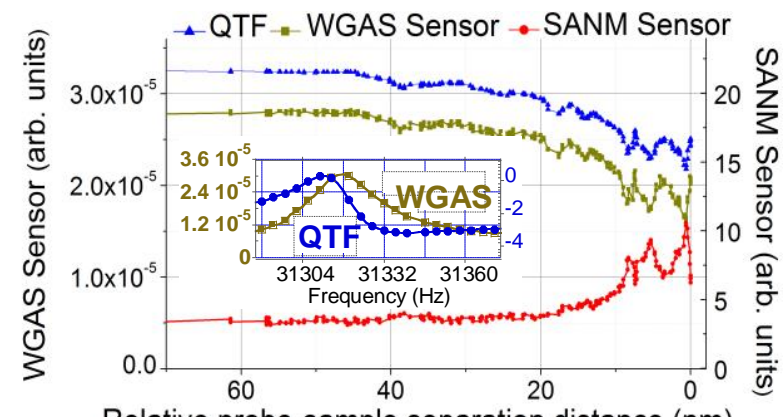

Relative probe-sample separation distance $(\mathrm{nm})$

Fig. 4 Approaching curves showing the correlation among the QTF's electrical admittance (top) and the acoustic WGAS (middle) and SANM signals (bottom). The inset shows the frequency response of the electrically driven QTF monitored by the QTF's admittance and WGAS; notice the discrepancy of the frequencies at which these signals peak.

In figures 2 and 3, the behavior of the SANM signal (that monitors the response from the mesoscopic film) has been contrasted with the conventional QTF electrical admittance. However the latter, while very sensitive, does not correlate directly with the mechanical motion of the QTF; $i$. $e$. the electrical response does not correlate exactly with the QTF mechanical motion. This is revealed by the inset in Fig. 4, which shows that the electrical resonance peak does not coincide with the mechanical resonance of the QTF (including its mounted tip). This is due to the inherent capacitance of the QTF that convolutes the measurement.
The WGAS signal instead correlates directly with the QTF mechanical motion; their frequency responses in fact superimpose each other. [11] Thus, when exact information of the QTF's mechanical motion state is needed (this would be relevant in friction phenomena studies), the use of the WGAS technique would be preferred. Figure 4 shows the existent correlation among the QTF, WGAS and SANM signal as the probe (tapered bare fiber probe) approaches an uncoated mica sample.

To gain further understanding of the SANM working principle, hydrodynamic measurements were performed by dipping a cleaved optical fiber (125 micrometers OD) into a water droplet placed on a mica sheet and using the setup shown in Fig.1. At each of the selected vertical positions (separated by 40 micrometer steps), the QTF's admittance and SANM's spectral responses were recorded; see Fig. 5. A dampening of the QTF's signal correlating with an increase in the acoustic amplitude is observed. In addition, a red shift of the resonance frequencies is observed in both the QTF and the SANM acoustic traces. At the last step, a probesample separation was still greater than 30 micrometers; hence, the possibility of the increase in acoustic signal resulted from a direct probe-sample contact can be ruled out. Instead, we argue that as the submersion depth of the probe increases, a greater amount of water is dragged by the probe, thus a stronger acoustic wave is built up.
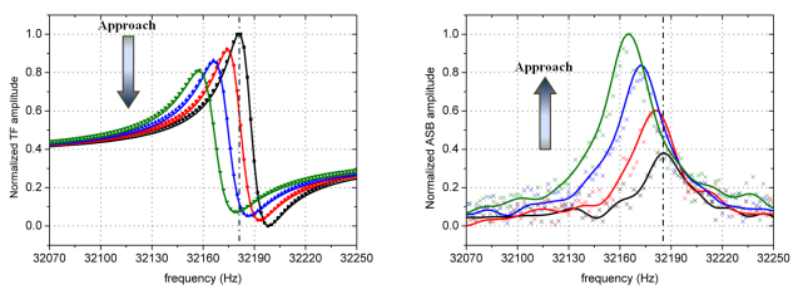

Fig. 5 Spectra of the QTF admittance and SANM acoustic signals recorded at different probe-sample distances (40 micrometer steps) while keeping the probe dipped into a water droplet.

For comparison purposes, another experiment was carried out by bringing the same cleaved fiber into the near proximity of a mica surface (starting at approximately less than 1 micrometer separation distance) without the presence of a water droplet. The ambient environment registered a $65 \%$ relative humidity at the time. The probe's vertical motion was controlled by a piezoelectric stage (Nano-OP65, Mad City Labs Inc.) The QTF admittance and SANM spectra were recorded at different probe-sample distances (each step $30 \mathrm{~nm}$ apart), which are shown in Fig 6. Notice the monotonic decrease of the tuning fork's vibration amplitude and a corresponding increase in the acoustic signal; similar to the experiment with the water droplet. However, the behavior of the changes in the resonance frequency peak is different. At the early stages of the approach the central frequency remains practically unchanged (first and second traces). However, a blue shift of the resonance frequency (increase in resonance frequency) is observed in the final region where the tip is estimated to be separated from the surface by a few nanometers (blue and green traces). This blue-shift is contrary to the red-shift 
characteristic of the hydrodynamic experiment described above.
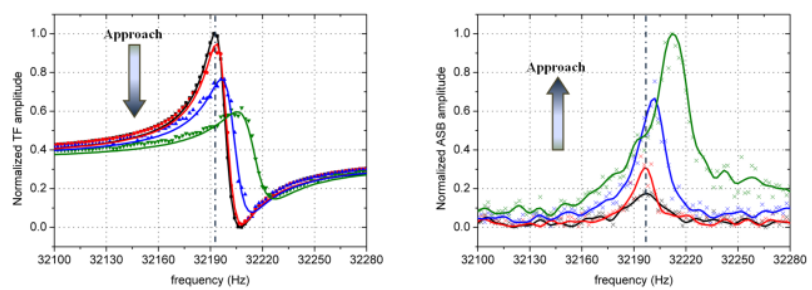

Fig. 6 Spectra of the QTF admittance and SANM acoustic signals recorded at different probe-sample distances (30 $\mathrm{nm}$ steps) during an approaching process.

\section{ANALYSIS}

Figures 2 and 3 clearly show that the lateral motion of the tapered metallic probe is affected even when the tip is positioned at a large distances $(\sim 30 \mathrm{~nm})$ from the goldcoated substrate (the position of the latter estimated from the tunnelling current measurement). Although long range electrostatic forces have been reported to affect the motion of atomic force microscopy (AFM) probes [12] their role in this case is less likely, given the fact that the stiffness of the QTF (spring constant $k \sim 20,000 \mathrm{~N} / \mathrm{m}$ ) is a few orders of magnitude greater than typical AFM probes $(k \sim \mathrm{N} / \mathrm{m})$. Electromagnetically induced shear forces on the probe caused by current dissipations (when a charged tip is moved parallel to a conducting material) are also expected to be negligible [13]. Alternatively, the concurrent detection of SANM acoustic signals (while the tunnel current sensor indicates no mechanical contact between the tip and the substrate) provides evidence of the existence of an adsorbed fluid layer (a medium where the acoustic waves are engendered by the oscillating probe, subsequently coupling into the substrate and reaching the SANM sensor) and its role as likely source of the shear forces acting on the probe. This finding is significant, as it sheds light onto the long standing questions about the origins of shear-forces. The existence of such adsorbed fluid layers is in fact widely accepted, particularly by the practitioners of dip pen nanolithography, in which the presence of a water meniscus around the tip is essential for the technique to work [14]. There exists a consensus that even at zero relative humidity, a mesoscopic water layer remains adsorbed to a solid substrate [15]. Accordingly, its presence becomes a plausible mean for the generation of acoustic waves while being shaken by a laterally oscillating probe and, reciprocally, affecting the motion of the probe. The hysteresis displayed by the acoustic SANM approaching/retraction traces (figures 2 and 3) further supports the role of the adsorbed fluid layer as the source of shear-forces, since during the retraction additional interactions may be caused by the substrate adhesive forces (or additional molecules adhered to the tip). Also, greater amount of material driven by the tip during the retraction would translate into a stronger acoustic signal. The latter is supported by the hydrodynamic experiment. Figure 5 indeed displays that as the probe gets progressively more immersed into the water droplet, the acoustic signal increases proportionally (despite a decreasing of the probe's oscillation amplitude). On the other hand, the fact that the resonance frequency increases as the tip approaches the substrate when the droplet is not present may reveal the substantial differences between bulk fluid (red-shift of the resonance frequency) and mesoscopic fluids (blue-shift of the resonance frequency) behaviour. However, controlled experiments that monitor the changes of the resonance under conditions that can rule out the possibility of probe-sample mechanical contact will be needed before reaching a solid conclusion.

Altogether, the acoustic-based experimental results presented here are significant for they provide evidence that an explanation of the origin shear force interactions (that affect the motion of an oscillating tip) does not necessarily require invoking a tip-substrate mechanical contact. The simultaneous acoustic detection of these interactions allows to suggest that the modification of the probe's motion is caused by its interaction with the mesoscopic fluid layer found adsorbed at the substrate surface.

\section{ACKNOWLEDGMENT}

A. L. R. thanks support from the "Faculty Enhancement Grant Program" at Portland State University, Portland, Oregon; and from the 2011 Milt Smith and Evelyn Kamback Fund, foundation account 2320222 .

\section{REFERENCES}

[1] Donald H. Buckley, "Surface effects in adhesion, friction, wear and lubrication" Elsevier Scientific Pub. Co. (1981).

[2] Horst Czichos, "Tribology: a systems approach to the science and technology of friction, lubrication, and wear," Elsevier Science (1978).

[3] Valentin L. Popov, "Contact mechanics and friction: physical principles and applications," Springer (2010).

[4] Fereydoon Family, H. G. E. Hentschel, and Y. Braiman, "Friction at the nanoscale." J. Phys. Chem. B 104, 3984 (2000).

[5] Enrico Gnecco, Sabine Maier, Oliver Pfeiffer, Alexis Baratoff, Roland Bennewitz, and Ernst Meyer, "Atomic-scale control of friction by actuation of nanometer-sized contacts," Science 313, 207 (2006).

[6] Yifei Mo, Kevin T. Turner, and Izabela Szlufarska, "Friction laws at the nanoscale," Nature 457, 1116 (2009).

[7] M. Cieplak, E.D. Smith, M.O. Robbins, "Molecular origins of friction: the force on adsorbed layers," Science 265, 1209 (1994).

[8] J. Krim, D.H. Solina, R. Chiarello, "Nanotribology of a Kr monolayer: a quartz crystal microbalance study of atomic-scale friction," Phys. Rev. Lett. 66, 181 (1991).

[9] K. Karrai and R. D. Grober, "Piezoelectric tip-sample distance control for near field optical microscopes," Appl. Phys. Lett. 66, 1842 (1995).

[10] A. La Rosa, L. Nan, R. Fernandez, X. Wang, and K. Prasanna, "Whispering-Gallery Acoustic Sensing," submitted.

[11] A. H. La Rosa, R. Nordstrom, X. Cui, J. McCollum, "The Ultrasonic/shear-force microscope: Integrating ultrasonic sensing into a near-field scanning optical microscope," Rev. Sci. Instrum. 76, 093707 (2005).

[12] Y. Sugimoto, P. Pou, M. Abe, Pavel Jelinek, R. Perez, S. Morita and O. Custance, "Chemical identification of individual surface atoms by atomic force microscopy, Nature 446, 64 (2007).

[13] E. Ayars, D. Aspnes, P. Moyer, and M.A. Paesler, "Proximal electromagnetic shear-forces," J. of Microscopy 196, 59 (1999).

[14] K. Salaita, Y. Wang and C. A. Mirkin, "Applications of dip-pen nanolithography," Nature Nanotechnology 2, 145 (2007).

[15] S. Rozhok, P. Sun, R. Piner, M. Lieberman, and Chad A. Mirkin, "AFM Study of Water Meniscus Formation between an AFM Tip and NaCl Substrate," J. Phys. Chem. B 108, 7814 (2004). 